\title{
ON THE POLYNOMIAL OF THE BEST APPROXIMATION TO A GIVEN CONTINUOUS FUNCTION*
}

\author{
BY J. SHOHAT (JACQES CHOKHATE)
}

1. A Theorem on Minimizing Polynomials. Let $f(x)$ and $p(x)$ be defined on a finite interval $(a, b) ; f(x)$ is bounded and integrable, $p(x)$ is integrable and not negative.

THEorem I.t If there exist two numbers $\alpha, \beta$ such that $a \leqq \alpha<\boldsymbol{\beta} \leqq b$, and such that

$$
\int_{c}^{d} p(x) d x>0
$$

whenever $\alpha \leqq c<d \leqq \beta$, then there exists one and only one polynomial of degree $\leqq n$ minimizing the integral

where

$$
I_{n k}=\int_{a}^{b} p(x)\left|f(x)-U_{n k}(x)\right|^{k} d x,
$$

$$
U_{n k}(x)=\sum_{i=0}^{n} u_{i k} x^{i}
$$

provided that $k>1$. If $k=1$, the proof of existence applies without change; and the approximating polynomial is unique, if $f(x)$ is continuous on $(a, b)$, and if

$$
\int_{c}^{d} p(x) d x>0
$$

whenever $a \leqq c<d \leqq b$.

The proof may be organized as follows.

* Presented to the Society, December 26, 1924. The author wishes to acknowledge with appreciation many helpful suggestions made by Professor D. Jackson in connection with this paper.

$\dagger$ Cf. G. Pólya, Sur un algorithme ..., Comptes Rendus, vol. 157 (1913), pp. 840-843; D. Jackson, On functions of closest approximation, Transactions of this Society, vol. 22 (1921), pp. 117-128, Note on a class of polynomials of approximation, ibid., vol. 22 (1921), pp. 320-326, $A$ generalized problem in weighted approximation, ibid., vol. 26 (1924), pp. 133-154, Note on the convergence of weighted trigonometric series, this Bulletin, vol. 29 (1923), pp. 259-263. 
2. Existence of a Minimizing Polynomial. The value of $n$ being regarded as fixed, take

$$
\delta=\frac{\beta-\alpha}{8 n^{2}} .
$$

For $y \pm \delta$ belonging to $(\alpha, \beta)$, we have

$$
\int_{y}^{y+\delta} p(x) d x, \quad \int_{y-\delta}^{y} p(x) d x \geqq h(\delta)=h_{n},
$$

since these integrals are continuous and positive functions of $y ; h_{n}$ depends on $n$ only. For an arbitrarily large $A>0$ we can find, according to Kirchberger,* certain $K_{i}$ such that any one of the inequalities $\left|u_{i k}\right|>K_{i}(i=0,1,2, \ldots, n)$ implies

$$
\begin{array}{r}
\max \left|U_{n k}(x)\right| \text { in }(\alpha, \beta)=\left|U_{n k}(\xi)\right|=M>2 \Phi+2 \sqrt[k]{A / h_{n}}, \\
\Phi=\max |f(x)| \text { in }(a, b), \quad \alpha \leqq \xi \leqq \beta .
\end{array}
$$

Using Markoff's theorem $\dagger$ and (1), we find, for $|x-\xi| \leqq \delta$,

$$
\begin{aligned}
&\left|U_{n k}(x)-U_{n k}(\xi)\right| \leqq|x-\xi| \cdot \frac{2 n^{2} M}{\beta-\alpha} \leqq \frac{M}{4}, \\
&\left|U_{n}(x)\right|>\frac{M}{2}>\Phi+\sqrt[k]{A / h_{n}}, \\
&\left|f(x)-U_{n k}(x)\right|>\sqrt[k]{A / h_{n}},
\end{aligned}
$$

whence it follows that $I_{n k}>A$, since at least one of the intervals $(\xi-\delta, \xi),(\xi, \xi+\delta)$ belongs to $(\alpha, \beta)$. Therefore we must take $\left|u_{i k}\right| \leqq K_{i}(i=0,1,2, \ldots, n)$, which proves the existence of a minimizing polynomial. We use the notation

(5) $\quad M_{n k}=\min I_{n k}=\int_{a}^{b} p(x)\left|f(x)-P_{n k}(x)\right|^{k} d x$.

* Ueber Tchebychefsche Annäherungsmethoden, Dissertation, Göttingen, 1902.

$\dagger$ Cf., e. g., M. Riesz, Eine trigonometrische Interpolationsformel, ..., JAHREsBericht DeR DeUtschen Mathematiker-Vereinigung, vol. 23 (1914), pp. 354-368; pp. 359-360. 
3. Uniqueness of the Solution. Case I: $k>1$. It can be easily shown that

$$
\left|\frac{1}{2}(x+y)\right|^{k}<\frac{1}{2}\left(|x|^{k}+|y|^{k}\right)
$$

if $k>1$ and $x \neq y$. Assuming the existence of two nonidentical solutions $\varphi_{1}(x)=f(x)-P_{n k}^{\prime}(x), \varphi_{2}(x)=f^{\prime}(x)-P_{n k}^{\prime \prime}(x)$, we get, using in (6) the function $\varphi_{3}(x)=\frac{1}{2}\left[\varphi_{1}(x)+\varphi_{2}(x)\right]$,

$$
\int_{a}^{b} p(x)\left|\varphi_{3}(x)\right|^{k} d x<\frac{1}{2} M_{n k}+\frac{1}{2} M_{n k}=M_{n k},
$$

which is impossible.

Case II: $k=1$. Proceeding as above, we get the impossible inequality (7), unless $\varphi_{1}(x) \varphi_{2}(x) \geqq 0$ for $a \leqq x \leqq b$. Evidently every function of the type

$$
\varphi_{3}(x)=h \varphi_{1}(x)+l \varphi_{2}(x)=\varphi_{1}(x)+l \psi(x),
$$

where $\psi(x)=\varphi_{2}(x)-\varphi_{1}(x), h, l>0, h+l=1$, is also a solution. Inasmuch as $\varphi_{1}(x)$ and $\varphi_{2}(x)$ can never have opposite signs, all roots of $\varphi_{3}(x)$ are the roots common to $\varphi_{1}(x)$ and $\varphi_{2}(x)$, or, what is the same, to $\varphi_{1}(x)$ and $\psi(x)$. Since $\psi(x)$ is a polynomial of degree $\leqq n$, we conclude:

If there exist two solutions $\varphi_{1}(x), \varphi_{2}(x)$, there exists necessarily a third one $\varphi_{3}(x)$, which has not more than $n$ roots in $(a, b)$. We shall prove that the last conclusion leads to a contradiction.*

Let the zeros of $\varphi_{3}(x)$ in $(a, b)$ be

$$
x_{1}<x_{2}<\ldots<x_{m} \quad(m \leqq n) .
$$

We have necessarily $m>0$; otherwise the function $\varphi(x)=\varphi_{3}(x)+\eta$ with a properly chosen $\eta$ gives $I_{n k}<M_{n k}$. Consider now two groups of the roots (9):

$$
z_{1}, z_{2}, \cdots, z_{m^{\prime}} ; \quad y_{1}, y_{2}, \cdots, y_{m^{\prime \prime}} \quad\left(m^{\prime}+m^{\prime \prime}=m\right),
$$

where the $z$ 's are the roots, if any, at which $\varphi_{3}(x)$ changes sign, and the $y$ 's are those at which it does not. Form the polynomial

* In the case that $f(x) \equiv x^{n+1}$, it was established in my Thesis that the number of roots is $n+1$. 


$$
\prod(x)= \pm \prod_{i=1}^{m^{\prime}}\left(x-z_{i}\right) \quad\left(\prod(x)=1 \text { for } m^{\prime}=0\right),
$$

where the sign \pm is chosen so that $\Pi(x) \varphi_{3}(x) \geq 0$ in $(a, b)$. Draw the curve $y=|\Pi(x)|$ and the line $y=\varepsilon$ with $\varepsilon>0$ sufficiently small. Project the points of intersection on the $x$-axis. Thus we get a set of points $Z$, made up of $m^{\prime}$ intervals

$$
\left(z_{i}-\delta_{i}^{\prime}, z_{i}+\delta_{i}^{\prime \prime}\right) \quad\left(i=1,2, \cdots, m^{\prime}\right),
$$

and a complementary set $K=b-a-Z$, such that

$$
\varepsilon=\max |\Pi(x)| \text { on } Z=\min |\Pi(x)| \text { on } K .
$$

It is evident that for $\varepsilon$ sufficiently small all maxima points of the curve and all $y$ 's are outside $Z$, and

$$
\int_{K} p(x) d x>3 \int_{Z} p(x) d x .
$$

The numbers $\varepsilon$ and $\delta_{i}^{\prime}, \delta_{i}^{\prime \prime}$ being fixed, we take $\delta>0$ so small that the set of points

$$
Y: \quad y_{j}-\delta \leqq x \leqq y_{j}+\delta \quad\left(j=1,2, \cdots, m^{\prime \prime}\right)
$$

is outside $Z$, and

$$
\begin{aligned}
& \int_{Y} p(x) d x<\frac{\varepsilon}{2 M}\left(\int_{K} p(x) d x-\int_{Z} p(x) d x\right), \\
& \int_{Y} p(x) d x<\int_{Z} p(x) d x,
\end{aligned}
$$

where $M$ is the maximum of $|\Pi(x)|$ in $(a, b)$. Since $\varphi_{3}(x)$ is continuous and has no zeros in

we have

$$
\begin{gathered}
K^{\prime}=b-a-Z-Y=K-Y, \\
\left|\varphi_{3}(x)\right| \geqq h^{\prime}>0 \quad \text { on } \quad K^{\prime} .
\end{gathered}
$$

We form now the function

$$
\varphi(x)=\varphi_{3}(x)-\eta \prod(x), \quad 0<\eta<h^{\prime} / M,
$$

and observe that 


$$
\left\{\begin{array}{l}
|\varphi(x)|=\left|\varphi_{3}(x)\right|-\eta\left|\prod(x)\right| \text { on } K^{\prime} \\
|\varphi(x)| \leqq\left|\varphi_{3}(x)\right|+\eta\left|\prod(x)\right| \text { on } Z+Y
\end{array}\right.
$$

whence, by combination with (12), (13), and (15), we get the desired contradiction:

$$
\int_{a}^{b} p(x)|\varphi(x)| d x<\int_{a}^{b} p(x)\left|\varphi_{3}(x)\right| d x=M_{n 1} .
$$

The existence proof gives incidentally the following corollary.

Corollary. To an arbitrarily large $A>0$ there correspond certain $K_{i}(i=0,1,2, \cdots, n)$ such that any one of the inequalities $\left|u_{i}\right|>K_{i}$ implies that

$$
\int_{a}^{b} p(x)\left|f(x)-\sum_{i=0}^{n} u_{i} x^{i}\right|^{k} d x>A,
$$

where $P(x)$ and $f(x)$ have the propertics specified, and $k \geqq 1$.

4. Tchebychef Approximating Polynomials. THEorem II. Let $f(x)$ be continuous in $(a, b), p(x) \geqq 0$, and

$$
\int_{\alpha}^{\beta} p(x) d x>0
$$

whenever $a \leqq \alpha<\beta \leqq b$. Then, for $k \rightarrow \infty$, the minimizing polynomial $P_{n k}(x)$ tends uniformly to the polynomial $T_{n}(f)$, of degree $\leqq n$, which gives the closest approximation to $f(x)$ in the interval $(a, b)$, in the sense of Tchebychef.

The proof is very similar to that given by G. Pólya, loc. cit. We assume, without loss of generality,

$$
\int_{a}^{b} p(x) d x=1,
$$

replacing, if necessary, $p(x)$ by $c p(x), c>0$ being properly chosen. We have evidently, taking $k>1$,

$$
\begin{aligned}
M_{n k} & =\min I_{n k}<E_{n}^{k}, \\
E_{n} & =\max \left|f(x)-T_{n}(f)\right| \text { for } a \leqq x \leqq b .
\end{aligned}
$$

If $a<\alpha<\beta<b$, and $\left|f(x)-P_{n k}(x)\right|$ attains its minimum in $(\alpha, \beta)$ at $x=z$, then we get, using (21), 


$$
\begin{gathered}
\left|P_{n k}(z)\right|<\frac{E_{n}}{\sqrt[k]{\int_{a}^{b} p(x) d x}}+\boldsymbol{\Phi}<\frac{E_{n}}{\int_{a}^{b} p(x) d x}+\boldsymbol{\Phi}, \\
\boldsymbol{D}=\max |f(x)| \text { in }(a, b) .
\end{gathered}
$$

We form now $n+1$ partial intervals $\left(\alpha_{i}, \beta_{i}\right)$ all belonging to $(a, b)$ and separated by segments of a certain length $\delta$, and we use (22). Since the right-hand member in (22) does not depend on $k$, we can follow the reasoning of G. Pólya and prove (a) the coefficients in $P_{n k}(x)$, considered as functions of $k$, are bounded, so that the sequence $\left(P_{n k}\right)$ admits one or several limiting polynomials; (b) all these limiting polynomials coincide with the polynomial of best approximation $T_{n}\left(f^{\prime}\right)$, which is known to be unique.

COROLLARY. The polynomial minimizing the integral

$$
\int_{a}^{b} p(x)\left|x^{n}+\ldots\right|^{k} d x
$$

tends for $k \rightarrow \infty$ uniformly to

$$
\frac{1}{B} \cos (n \arccos y), \quad y=\frac{2 x-a-b}{b-a},
$$

for every $p(x)$ satisfying the conditions of Theorem II, where $B\left(=2^{\left.2 n-1 /(b-a)^{n}\right)}\right.$ is the coefficient of $x^{n}$ in $\cos (n \operatorname{arc} \cos y)$.

In fact, $x^{n}-(1 / B) \cos (n \operatorname{arc} \cos y)$ is the polynomial of degree $\leqq n-1$ giving the best approximation to $x^{n}$ in $(a, b)$.

The results given above hold if we replace polynomials by trigonometric sums, provided $p(x)$ and $f(x)$ are periodic functions.

The University of Chicago 